\title{
Chris Arthur, Hummingbirds Between the Pages
}

\author{
Marion Naugrette-Fournier
}

\section{(2) OpenEdition}

\section{Journals}

\section{Édition électronique}

URL : https://journals.openedition.org/etudesirlandaises/7388

DOI : 10.4000/etudesirlandaises. 7388

ISSN : 2259-8863

\section{Éditeur}

Presses universitaires de Caen

\section{Édition imprimée}

Date de publication : 14 novembre 2019

Pagination : 155-157

ISBN : 978-2-84133-945-7

ISSN : 0183-973X

\section{Référence électronique}

Marion Naugrette-Fournier, "Chris Arthur, Hummingbirds Between the Pages », Études irlandaises [En ligne], 44-1 | 2019, mis en ligne le 14 novembre 2019, consulté le 14 novembre 2022. URL : http:// journals.openedition.org/etudesirlandaises/7388; DOI : https://doi.org/10.4000/etudesirlandaises. 7388

\section{(c) (i) (2)(2)}

Creative Commons - Attribution - Pas d'Utilisation Commerciale - Partage dans les Mêmes Conditions 4.0 International - CC BY-NC-SA 4.0

https://creativecommons.org/licenses/by-nc-sa/4.0/ 
à travers leurs propres déclarations dans la presse ou dans leurs mémoires. L'aspect le plus remarquable est sans doute la capacité de l'auteur à souligner la perméabilité entre ces différents mouvements, mais aussi à restituer les nuances et les tensions entre eux, tout en adoptant une perspective internationale qui englobe le contexte politique aux États-Unis, en France ou en Allemagne.

Le contenu de cette seconde édition est inchangé par rapport à la première édition de 2007, à l'exception d'une préface de cinq pages ajoutée par l'auteur, qui saisit l'occasion de revenir sur son propre travail avec onze années de recul. Il se livre à une évaluation critique de ses propres conclusions de l'époque, soulignant à la fois celles qui résistent à l'épreuve du temps et celles qui ont quelque peu perdu de leur pertinence, tout en proposant de nouvelles interprétations possibles. Il ne manque pas de revenir également sur quelques controverses nées à la suite de la publication de la première édition, ce qui apporte un éclairage tout à fait intéressant sur l'ouvrage.

Charlotte BARCAT

\section{Chris Arthur, Hummingbirds Between the Pages, Columbus, Mad Creek Books - The Ohio State University, 2018, 245 p.}

«L'essai, ce n'est pas mon genre.» C'est précisément contre cette petite phrase si souvent entendue que l'essayiste nord-irlandais Chris Arthur part en croisade dans l'un de ses recueils d'essais les plus récents, au titre à la fois poétique et sibyllin, Hummingbirds Between the Pages. Chris Arthur n'en est pas à son coup d'essai en la matière, puisqu'il a déjà publié plusieurs volumes d'essais acclamés par la critique, dont la trilogie Irish Nocturnes (1999), Irish Willow (2002) et Irish Haiku (2005), que viennent ensuite compléter Irish Elegies (2009), On the Shoreline of Knowledge (2012) et Reading Life (2017). Arthur, qui contrairement à beaucoup d'autres écrivains de sa génération écrit exclusivement des essais (à l'exception de certaines échappées poétiques au début de sa carrière), renouvelle et dépoussière le genre, s'insurgeant contre sa réputation parfois peu attrayante, «a form of belleslettres whose day is done» (p. 237).

Chris Arthur, tout au long de ces treize essais, réaffirme avec force et érudition la dimension de l'intime qui est au cœur de ce genre littéraire, en nous livrant ses «colibris métaphoriques» («my metaphorical hummingbirds», introduction, p. x; je traduis), qui ont provoqué chez lui l'interrogation et lui ont laissé entrevoir la possibilité de connections inconnues, insoupçonnées, et de strates temporelles vertigineuses. La plupart de ces colibris prennent souvent la forme banale d'objets du quotidien, comme une pendule sans charme, de vieilles photographies, ou bien des traverses de chemin de fer («sleepers», p. 34), que son père utilisait pour alimenter le feu familial. Comment ces objets ordinaires revêtent-ils alors aux yeux de l'essayiste les attributs chatoyants des colibris, qui avaient tant émerveillé le jeune Chris Arthur lors de sa visite au zoo de Londres lorsqu'il avait huit ans? 
But all of them are hummingbird-like in the way they offer unexpected challenges to the mundane scales by which we measure things; they suggest new calibrations in terms of meaning, connection, and significance as surely as the London birds suggested a different scale for color. (introduction, p. $\mathrm{x}$ )

Sans pour autant posséder l'iridescence ou la palette chromatique des colibris, ces objets du quotidien ont en commun avec ces oiseaux la qualité d'avoir attiré le regard de l'essayiste, qui, tel le chiffonnier de Baudelaire, ramasse et collectionne dans ses essais les fragments de la réalité ordinaire, lui suggérant un réseau de significations et de ramifications qui vont au-delà de leur simple statut d'objets: "[...] they have left me feeling that I'm standing on the frontier of the ordinary being given glimpses of the extraordinary dimensions it contains" (ibid.).

La démarche d'Arthur n'est pas sans évoquer celle du naturaliste ou du biologiste, qui «presse» (voir ce motif en introduction, p. ix, $x, x i .$. ) ses spécimens entre les pages de ses ouvrages, ses «hummingbirds», qu'ils soient londoniens, nord-irlandais, écossais, voire même asiatiques, telle cette statuette de Bouddha achetée en Écosse en 1983, et qui fait l'objet d'un texte fascinant, "The Walking Buddha Beckons » (p. 24-33). Il n'est donc guère surprenant que le premier essai du recueil soit un hommage à Darwin, intitulé «Darwin's Fox» (p. 1-12), qui est tout à fait représentatif de la méthode d'investigation qu'adopte Chris Arthur face aux échantillons du réel qu'il trouve (mais sélectionne en même temps). Dans ce texte, il relate ainsi comment Darwin tua un renard (Canis fulvipes), lorsqu'il débarqua sur l'île de San Pedro le 6 décembre 1834 lors de son expédition à bord du Beagle. À partir de cet incident, Arthur développe une grille de possibilités qui auraient pu empêcher que cette rencontre fatale entre Darwin et le renard se produise:

If a seabird had faltered from exhaustion, attracting the fox to easy prey; if Darwin had taken a different route as he explored the island; if a storm had made it impossible to land; if the Beagle's crew had been laid low with dysentery; if he'd sneezed as he swung his geological hammer, this moment would never have happened. (p. 6-7)

Au fil des essais qui constituent Hummingbirds Between the Pages, Chris Arthur choisit avec la curiosité attentive au monde qui l'entoure typique du collectionneur des objets ou des moments, des rencontres parfois (telles que celle de Darwin et du renard), qu'il va ensuite "épingler» entre les pages de son livre, car ils suscitent en lui l'interrogation sur leurs conditions de possibilité: que représentent-ils? Aurait-il pu en être autrement? Quelles sont leurs implications, voire leurs répercussions sur le passé et le futur? Ce questionnement métaphysique occasionné par les incidents du réel cache cependant une angoisse plus profonde chez l'essayiste - la peur de la mort. «Glass» (p. 59-64) évoque la première rencontre de la fille de Chris Arthur avec la mort, sous la forme d'une mésange bleue venant se fracasser mortellement à la fenêtre de la chambre. Cet événement, dans sa simplicité et sa brutalité, incarne aux yeux de l'essayiste de manière métonymique la fatalité de la mort, qui peut survenir à n'importe quel instant - à tout moment la dureté d'une vitre invisible peut venir arrêter la course de la vie. De même, la pendule (p. 72 ; voir l'essai 
intitulé «(Un)sentimental Timekeeping», p. 65-74) ayant appartenu à la mère d'Arthur dans les dernières années de sa vie, passées dans une maison de retraite en Irlande du Nord, tandis que son fils faisait les allers-retours depuis l'Écosse en ferry, lui évoque une capsule temporelle qui aurait emmagasiné les derniers jours de sa mère, sa présence et son absence. Cette pendule sert à l'essayiste de vanitas, et lui rappelle l'inexorabilité de la mort et la nécessité de ne pas perdre de temps, «as much an aide-mémoire for finitude as a practical chronometer» (p. 72; voir également, concernant le motif isotopique du memento mori, "Death and the Maiden», p. 130 et «The Archaeology of Days», p. 176).

À la lecture de ces "colibris métaphoriques», pour reprendre l'expression d'Arthur, on est parfois tenté de lui reprocher une certaine tendance à l'exagération, ou de prendre les choses par le petit bout de la lorgnette, ce qu'il nie d'ailleurs: "I've tried to resist the theatrics of exaggeration; tried not to make mountains out of molehills» (p. 62). L'écriture est parfois précieuse, tant elle vise à l'exactitude et à la minutie du détail, voire à la limite de la pédanterie, tant elle est savante dans ses moindres expressions. Mais, petit à petit, l'œil du lecteur s'habitue à ce constant changement d'échelle entre microcosme et macrocosme, et finit par se délecter du raffinement de l'écriture de l'essayiste, qui prend le temps d'analyser les phénomènes du réel qui nous entoure, dans toute leur infinie diversité. Dans son épilogue intitulé «Thirty-Six Ways of Looking at an Essay» (p. 237-242), d'après les Cent vues du mont Fuji d'Hokusai, Arthur décrit les modalités qui selon lui caractérisent le genre de l'essai. Nous n'en retiendrons ici que la première: «An essay richly complicates the commonplace, revealing mazes of meaning coiled within the mundane» (p. 238).

\section{Marion NAUgrette-Fournier}

\section{Hedda Friberg-Harnesk, Reading John Banville through Jean Baudrillard, Amherst - New York, Cambria Press, 2018, 222 p.}

Hedda Friberg-Harnesk sheds new light on the fiction of John Banville thanks to an original theoretical frame: her perceptive use of Baudrillard's orders of simulacra engages the reader in a stimulating critical conversation with the unstable and uncertain worlds and beings of Banvillean fiction. She explores nine of John Banville's works published between 1997 and 2015, seven novels (The Untouchable, Eclipse, Shroud, The Sea, The Infinities, Ancient Light, and The Blue Guitar) and two plays (God's Gift and Love in the Wars), favouring his later works over earlier literary productions, finding there a more acute, more accomplished sense of the "simulation of an absent reality" (p. 7), which to Baudrillard characterises our capitalist society. The author's eloquent, thought-provoking study grants the reader a deeper insight into Banville's bewitching "territory of mercurial instability" (p. 1). 